\title{
ERM/Rho protein expression in ductal breast cancer: a 15 year follow-up
}

\author{
Agnieszka Halon • Piotr Donizy • Pawel Surowiak • \\ Rafal Matkowski
}

Accepted: 31 January 2013 / Published online: 19 February 2013

(C) The Author(s) 2013. This article is published with open access at Springerlink.com

\begin{abstract}
Purpose The aim of this study was to examine the expression of ERM (ezrin, moesin) and Rho (RhoA, RhoB and $\mathrm{Cdc} 42$ ) proteins in breast cancer (BC) patients and to investigate the relationship between the sub-cellular localisation of these proteins and clinicopathological characteristics and patient survival.

Methods The expression and specific sub-cellular distribution of the ERM/Rho proteins was analysed by immunohistochemistry in a homogeneous group of 85 stage II ductal $\mathrm{BC}$ patients with a follow-up of 15 years.

Results Enhanced immunoreactivity of all analysed proteins was found to be associated with the presence of lymph node metastases (ezrin, $P=0.047$, moesin, $P=0.038$, RhoA, $P=$ 0.024, RhoB, $P=0.004$ and $\mathrm{Cdc} 42, P=0.047$ ). Nuclear localisation of ezrin was found to correlate with the presence of lymph nodes metastases $(P=0.004)$ and with histological de-differentiation $(P=0.015)$. In contrast, we found that the nuclear topography of RhoA and $\mathrm{Cdc} 42$, and the perinuclear localisation of RhoB, were strongly associated with a lack of
\end{abstract}

\footnotetext{
A. Halon $(\square) \cdot$ P. Donizy

Department of Pathomorphology and Oncological Cytology,

Wroclaw Medical University, ul. Borowska 213,

50-556 Wroclaw, Poland

e-mail: ahalon2@gmail.com

P. Surowiak

Department of Histology and Embryology,

Wroclaw Medical University, Chalubinskiego 6a,

50-356 Wroclaw, Poland

R. Matkowski

Department of Oncology and Division of Surgical Oncology,

Wroclaw Medical University, pl. Hirszfelda 12,

53-413 Wroclaw, Poland

R. Matkowski

Lower Silesian Oncology Centre, pl. Hirszfelda 12,

53-413 Wroclaw, Poland
}

nodal metastases ( $P=0.008, P=0.048, P=0.001$, respectively), whereas a decreased reactivity of RhoA in the stromal compartment of $\mathrm{BC}$ tumours was associated with the presence of lymph node metastases $(P=0.011)$. No relationship was observed between ERM/Rho protein expression and oestrogen receptor (ER), progesterone receptor (PgR) or HER-2 reactivity in the $\mathrm{BC}$ cells. Also, ERM/Rho protein expression did not predict patient survival, but RhoB overexpression in the stromal compartment of the tumours was found to be associated with a better prognosis $(P=0.0106)$. Conclusions The ERM/Rho immunoprofile and the assessment of its specific sub-cellular localisation may be instrumental for the prediction of lymph node metastases in ductal $\mathrm{BC}$ patients.

Keywords ERM proteins $\cdot$ Rho proteins $\cdot$ Breast cancer . Immunohistochemistry

\section{Introduction}

Breast cancer (BC) continues to be a major cause of morbidity and mortality, and it is one of the most common causes of cancer death in women world-wide. In 2008, more than 1.38 million new $\mathrm{BC}$ cases were diagnosed, leading to the death of approximately 500,000 individuals [1]. The standard method of $\mathrm{BC}$ treatment is multimodality management: endocrine therapy, surgery, radiotherapy, chemotherapy and molecularly targeted therapy. Conventional prognostic and predictive factors include disease stage (tumour size, nodal status and presence of distant metastases) [2], expression of oestrogen and progesterone receptors (ER and PgR, respectively) [3], immunohistochemical/molecular status of HER-2 [4], and Ki67 expression. Detailed gene expression analyses have led to the recognition of five fundamentally different subtypes of BC. However, since gene expression analysis of BC tumour material is not always feasible in clinical practice, a simplified 
classification system was recently recommended by the St. Gallen International Expert Consensus group. The main elements of this classification system include an immunohistochemical evaluation of ER and PgR status, assessment of over-expression and/or genomic amplification of HER-2, and the Ki-67 labelling index, which is a marker for cellular proliferation [5].

Ezrin, radixin and moesin, collectively known as ERM proteins, exhibit tissue-specific expression patterns. Ezrin expression is predominantly observed in epithelial cells, whereas moesin is expressed in endothelia, and radixin in hepatocytes [6]. ERM proteins function as structural and regulatory crosslinkers organising membrane-cytoskeleton-associated complexes. As such, they play a crucial role in the control of cell morphology. They also regulate a wide range of cellular activities including polarity, motility, adhesion and survival, all of which are associated with cancer development and progression [7]. ERM proteins are capable of interacting with several cellular molecules that are strongly associated with tumour progression, including CD43, CD44, ICAM-1, ICAM-2, EGFR and MET [7, 8]. Several recent studies have reported over-expression of ezrin as a marker of poor outcome in a variety of human cancers [9-12]. The significance of moesin immunoreactivity in tumour tissues, however, still remains to be established.

RhoA, RhoB and Cdc42 are members of the Ras homologous (Rho) family of low molecular weight GTP-binding proteins [13], and they act as major regulators of signal transduction pathways affecting remodelling of the actin cytoskeleton. Initial studies investigating the role of RhoA in cellular development revealed that over-expression of RhoA stimulates actin polymerisation through the activation of diaphanousrelated formins (DRFs) and Rho-activated kinases (ROCKs). A cooperation of RhoA, DRFs and ROCKs was found to induce the formation of stress fibres, which are necessary for maintaining cellular polarity and the proper shape of cells [14]. The specific functions of RhoB have not been described in detail yet, but experimental studies have shown that RhoB is down-regulated in human tumours and that its expression is inversely related to tumour progression [15]. In addition, it was found that $\mathrm{Cdc} 42$ is involved in increased cellular motility via participation in the filopodia formation process [16].

The significance of ERM and Rho protein immunoreactivity in breast carcinogenesis and the impact on long-term survival has so far remained unclear. In this study, we investigated the prognostic value of ERM (ezrin, moesin) and Rho (RhoA, RhoB and Cdc42) protein expression by immunohistochemical analyses in 85 patients with stage II ductal BC. We assessed the relationship between the sub-cellular localisation of these proteins in conjunction with the clinical characteristics of the patients studied. Additionally, we assessed the correlation between ERM/Rho reactivity in BC specimens and the status of receptors for the female sex hormones (i.e.,
ER and PgR) and HER-2. Since the treatment protocol for patients with stage II BC is still under debate, it is imperative to establish new immunohistochemical markers for the stratification of patients with stage II BC and, thus, to facilitate personalised treatment tailored to tumour aggressiveness.

\section{Material and methods}

\subsection{Patients}

Tissue samples were obtained from 85 patients treated radically for stage II ductal breast cancer (BC), diagnosed between 1993 and 1994 in the Lower Silesian Oncology Centre in Wroclaw, Poland. The median age of the patients was 55.6 years. The patients were selected based on the availability of tissues. They were not stratified for preoperative or pathological prognostic factors. All patients underwent surgery (Madden mastectomy) with or without adjuvant treatment. Cancer-specific overall survival (CSOS) and disease-free survival (DFS) rates were established for all patients. The total number of patients included was stipulated by the single series performed by our institution, the follow-up period of 15 years, and the highly homogeneous characteristics of the tumours selected (ductal invasive breast cancer, G2 and G3, clinical stage II according to UICC, Madden mastectomy). Also, the patients were relatively young, which is due to the fact that the average life expectancy and healthy life span in Poland in the period 1993-1994 were lower than those in e.g. Western Europe. Detailed characteristics of the patient cohort are listed in Table 1. The study was approved by the Institutional Review Board of the Wroclaw Medical University, Poland.

\subsection{Tumour samples}

Tumour specimens were fixed in $10 \%$ buffered formalin and embedded in paraffin. All haematoxylin and eosin stained sections were examined by two pathologists. Due to the absence of a population-based BC screening at the time this study was initiated, the size of the primary tumours was different from the detriment of the value of other, e.g. European countries. The median tumour size was also determined by the inclusion of a homogeneous group of clinical stage II BC (see above). Tumour stages were assessed according to the TNM classification system [17]. The tumour grades were estimated according to the Bloom-Richardson protocol, with the Elston and Ellis [18] modification (Table 1).

\subsection{Immunohistochemistry}

Immunohistochemical analyses were performed retrospectively on tissue samples collected for routine diagnostic 
Table 1 Patient and tumour characteristics

\begin{tabular}{|c|c|}
\hline Characteristics & No. $(\%)^{\mathrm{a}}$ \\
\hline All patients & $85(100)$ \\
\hline \multicolumn{2}{|l|}{ Age (mean 55.6 \pm 10.7 ; median: 55 ) } \\
\hline \multicolumn{2}{|l|}{ Menopause } \\
\hline Premenopausal & $27(31.8)$ \\
\hline Postmenopausal & $58(68.2)$ \\
\hline \multicolumn{2}{|l|}{ Histology } \\
\hline Invasive ductal $\mathrm{BC}$ & $85(100)$ \\
\hline \multicolumn{2}{|l|}{ TNM stage according to UICC } \\
\hline II A & $34(40)$ \\
\hline II B & $51(60)$ \\
\hline \multicolumn{2}{|l|}{ Tumour size in $\mathrm{mm}(\mathrm{pT})$ : mean $31 \pm 12.2$; median: 30} \\
\hline \multicolumn{2}{|l|}{ Nodal metastases $(\mathrm{N})$} \\
\hline $\mathrm{N}(-)$ & $49(57.7)$ \\
\hline $\mathrm{N}(+)$ & $36(42.3)$ \\
\hline \multicolumn{2}{|l|}{ Grading } \\
\hline 2 & $61(72)$ \\
\hline 3 & $24(28)$ \\
\hline \multicolumn{2}{|l|}{ Therapy $^{\mathrm{b}}$} \\
\hline Tamoxifen & $49(57.7)$ \\
\hline Cyclophosphamide/Metotrexate/5-Fluorouracil & $23(27.1)$ \\
\hline Anthracyclines & $1(1.2)$ \\
\hline Without adjuvant chemotherapy & $61(71.8)$ \\
\hline Radiotherapy & $37(43.5)$ \\
\hline \multicolumn{2}{|l|}{ ER status } \\
\hline Negative & $22(25.9)$ \\
\hline Positive & $63(74.1)$ \\
\hline \multicolumn{2}{|l|}{ PgR status } \\
\hline Negative & $22(25.9)$ \\
\hline Positive & $63(74.1)$ \\
\hline \multicolumn{2}{|l|}{ HER-2 status } \\
\hline Negative $(0,1+, 2+)$ & $66(77.6)$ \\
\hline Positive $(3+)$ & $19(22.4)$ \\
\hline
\end{tabular}

${ }^{\text {a }}$ Percentages in the groups may not sum to $100 \%$ due to rounding off

${ }^{\mathrm{b}}$ Some patients received more than one special treatment

purposes. Formalin-fixed, paraffin embedded tissue sections were freshly prepared $(4 \mu \mathrm{m})$. Immunohistochemistry was performed as previously described [19-21] using the following antibodies diluted in Antibody Diluent, Background Reducing (DakoCytomation, Poland): anti-ezrin (1:150, clone 4A5 mouse monoclonal, Chemicon International, Billerica, USA), anti-moesin (1:100, Sc-6410, goat polyclonal, Santa Cruz Biotechnology, Santa Cruz, USA), anti-RhoA (1:100, Sc-179, rabbit polyclonal, Santa Cruz Biotechnology), antiRhoB (1:100, Sc-180, rabbit polyclonal, Santa Cruz Biotechnology) and anti-Cdc42 (1:50, Sc-87, rabbit polyclonal, Santa Cruz Biotechnology). For the detection of the oestrogen receptor, an optimally pre-diluted mouse monoclonal antibody (clone 1D5, DakoCytomation, Denmark) was used, and for the detection of the progesterone receptor, an optimally pre-diluted mouse monoclonal antibody (clone PgR636, DakoCytomation, Denmark) was used. For HER-2 detection, a semi-quantitative diagnostic immunohistochemical test was used (HercepTest ${ }^{\mathrm{TM} K i t,}$ K5207, DakoCytomation, Denmark). The tissue sections were incubated with antibodies for $1 \mathrm{~h}$ at room temperature. Subsequent incubations involved biotinylated antibodies (15 min, room temperature) and a streptavidinbiotinylated peroxidase complex (15 $\mathrm{min}$, room temperature) (LSAB+, HRP, DakoCytomation, Poland). NovaRed (Vector Laboratories, UK) was used as a chromogen (10 min, at room temperature). All sections were counterstained with Meyer's haematoxylin. In each case control reactions were included, in which the specific antibody was substituted by a Primary Mouse Negative Control (DakoCytomation, Poland).

\subsection{Evaluation of immunohistochemical reaction intensities}

The intensities of the immunohistochemical reactions were estimated independently by two pathologists. In order to evaluate the expression of the proteins analysed, a semiquantitative scale of the ImmunoReactive Score (IRS), with the author's own modifications, was applied [22], in which the intensity of the colour reaction and the percentage of positive cells were both taken into account. The final, integrated scores ranged from 0 to 12 . The author's IRS modification only involved the percentage of positive cells. This is presented in Table 2. Cases with expression scores ranging between 0 and 2 in the IRS scale were considered negative. For each of the analysed proteins (except moesin), next to a predominant cytoplasmic localisation, additional sub-cellular localisations were observed. Ezrin was found to display both membranous and nuclear localisations. RhoA and $\mathrm{Cdc} 42$ were also found to be located in the nuclei of the cells, and RhoB was found to be located in both the cell membrane and the perinuclear zone. In addition, all the analysed proteins were found to be present in the stromal compartments of the tumours and in its blood vessels. A three-step scale (SS, stromal score; BVS, blood vessel

Table 2 Procedure for evaluation of ERM and Rho protein expression

IRS (ImmunoReactive Score) modified by the authors ${ }^{\mathrm{a}}$

\begin{tabular}{llll}
\hline Percentage of positive cells & Points & Intensity of reaction & Points \\
\hline No positive cells & 0 & No reaction & 0 \\
$<25 \%$ positive cells & 1 & Weak colour reaction & 1 \\
$25-50 \%$ positive cells & 2 & Moderate intensity & 2 \\
$51-75 \%$ positive cells & 3 & Intense reaction & 3 \\
$>75 \%$ positive cells & 4 & &
\end{tabular}

${ }^{\mathrm{a}}$ IRS score (ImmunoReactive Score) according to Remmele et al. [22] modified by the authors 
score), including the intensity of the colour reaction and the percentage of stained tissue or blood vessels (SS or BVS 0: no reaction; 1 : weak reaction; 2 : moderately positive reaction; 3 : strongly positive reaction), was used for the evaluation of stromal and blood vessel reactivity.

The evaluation of oestrogen and progesterone receptor expression was performed using standard methods. The staining intensity ( $0-3$ scale) and proportion of positive cells ( $0-5$ scale) were reported, and the Allred score that combines the two was calculated. The HER-2 status was evaluated using a FDA-approved scoring system of $0,1+, 2+$ and $3+(0$ : no immunostaining; $1+$ : weak immunostaining, less than $30 \%$ of the tumour cells; $2+$ : complete membranous reactivity, either uniform or weak in at least $10 \%$ of the tumour cells; $3+$ : uniform intense membranous staining in at least $30 \%$ of the tumour cells).

\subsection{Statistical analyses}

Statistical analyses were performed using the Statistica 9.1 software package (StatSoft Inc., Tulsa, OK, USA). Diseasefree survival (DFS) was defined as the time between the primary surgical treatment and date of relapse or death, whichever occurred first. DFS was censored at the last follow-up for patients who survived without disease recurrence. Cancer-specific overall survival (CSOS) was defined as the time between the primary surgical treatment and cancer-associated death, and was censored at the last follow-up for surviving patients. The $\chi^{2}$ and Spearman rank correlation were used to analyse associations between ERM/Rho protein expression parameters and clinicopathological parameters. The overall survival rate was estimated by the Kaplan-Meier method and the log-rank test. $P$ values $<0.05$ were considered statistically significant.

\section{Results}

3.1 Ezrin and moesin immunostaining in breast cancer
specimens

Ezrin protein expression was detected in all breast cancer (BC) cases with a mean IRS of 8.88 . The dominant subcellular localisation of ezrin was cytoplasmic (Fig. 1a). Coexpression of ezrin in the cellular membrane (20 cases, $23.53 \%$ ) (Fig. 1b) and the nucleus (27 cases, $31.76 \%$ ) (Fig. 1c) was also observed. Immunostaining of moesin revealed only a cytoplasmic reaction with a mean IRS of 3.69 (Fig. 1d). Interestingly, microscopic analyses also revealed ezrin and moesin expression in the stromal compartment of the BC tumour specimens and in its blood vessels (Table 3).

\subsection{RhoA, RhoB and Cdc42 immunostaining in breast cancer specimens}

RhoA, RhoB and $\mathrm{Cdc} 42$ protein expression was detected in all breast cancer cases (Table 3). The dominant sub-cellular
Fig. 1 Immunohistochemistry of ezrin and moesin proteins. a cytoplasmic localisation of ezrin (ImmunoReactive Score (IRS) $12, \times 200$, haematoxylin); b membranous localisation of ezrin (IRS 12, ×100, haematoxylin); c nuclear localisation of ezrin (IRS $8, \times 100$, haematoxylin); $\mathbf{d}$ cytoplasmic localisation of moesin (IRS 9, ×100, haematoxylin)
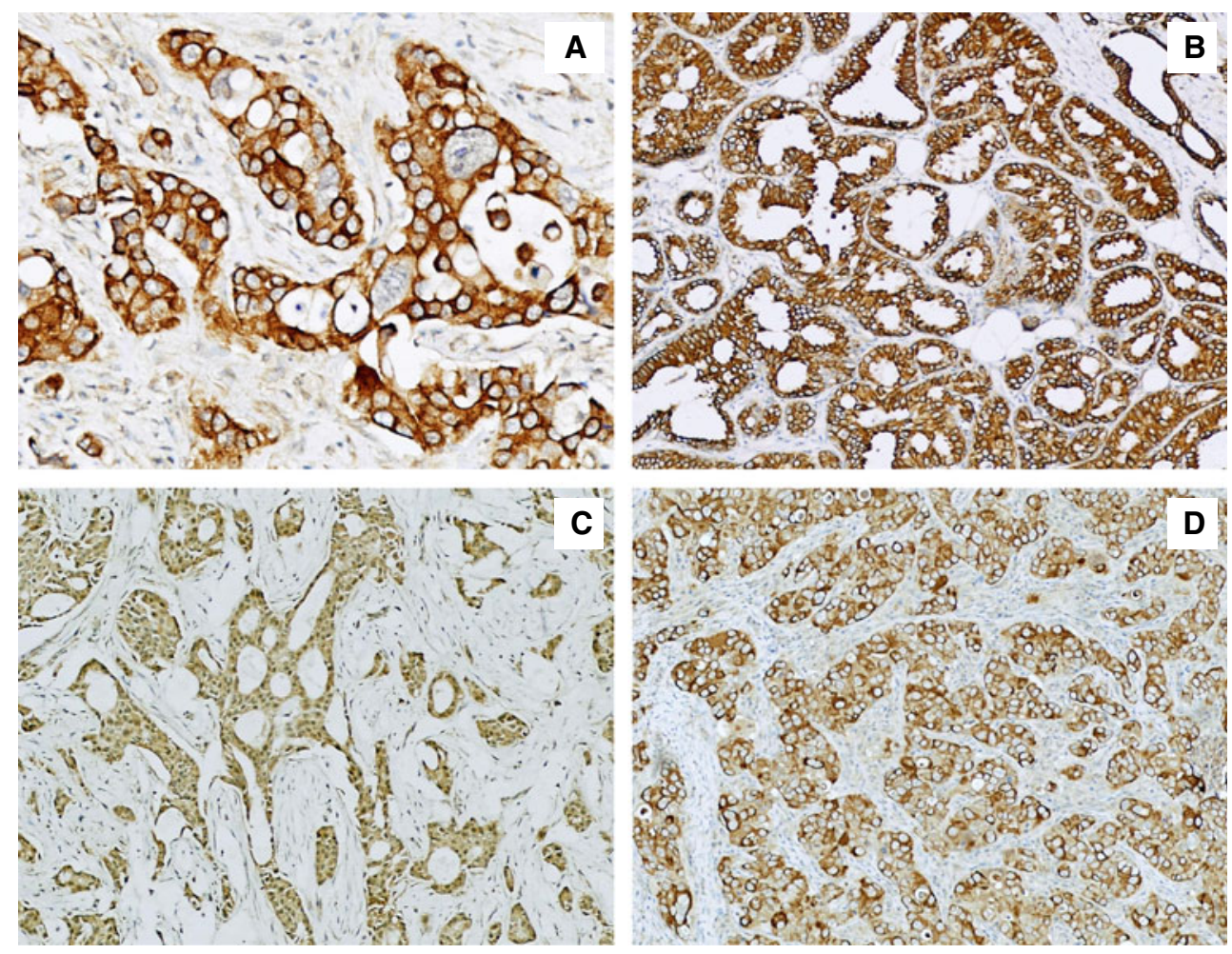
Table 3 Characteristics of ERM and Rho protein expression in the tumour and stromal compartments in breast cancer cases

${ }^{\mathrm{a}}$ Mean value and standard deviation

Parameters which are commented in text are underlined with bold text

\begin{tabular}{|c|c|c|c|c|c|}
\hline \multirow[t]{2}{*}{ Parameters of expression } & \multicolumn{5}{|c|}{ Number of patients (percentage) } \\
\hline & EZRIN & MOESIN & RhoA & RhoB & $\mathrm{Cdc} 42$ \\
\hline \multicolumn{6}{|l|}{ Quantitative parameters } \\
\hline Lack of expression $($ IRS $\leq 2)$ & $0(0)$ & $23(27.1)$ & $0(0)$ & $0(0)$ & $0(0)$ \\
\hline Positive reactivity (IRS > 2) & $85(100)$ & $62(72.9)$ & $85(100)$ & $85(100)$ & $85(100)$ \\
\hline$\%$ of positive cells ${ }^{\mathrm{a}}$ & $3.71 \pm 0.484$ & $2.44 \pm 1.220$ & $3.56 \pm 0.544$ & $3.54 \pm 0.547$ & $3.18 \pm 0.99$ \\
\hline Intensity of reaction ${ }^{\mathrm{a}}$ & $2.38 \pm 0.511$ & $1.45 \pm 0.592$ & $1.89 \pm 0.489$ & $2.09 \pm 0.503$ & $1.78 \pm 0.68$ \\
\hline IRS Score ${ }^{\mathrm{a}}$ & $8.88 \pm 2.51$ & $3.69 \pm 2.65$ & $6.74 \pm 2.09$ & $7.42 \pm 2.25$ & $5.96 \pm 3.32$ \\
\hline \multicolumn{6}{|c|}{ Subcellular topography in cancer cells } \\
\hline Cytoplasmic & $85(100)$ & $62(72.9)$ & $85(100)$ & $85(100)$ & $85(100)$ \\
\hline Membranous & $20(23.5)$ & $0(0)$ & $0(0)$ & $14(16.5)$ & $0(0)$ \\
\hline Nuclear & 27 (31.8) & $0(0)$ & $70(82.4)$ & $0(0)$ & $6(7.1)$ \\
\hline Perinuclear & $0(0)$ & $0(0)$ & $0(0)$ & $24(28.2)$ & $0(0)$ \\
\hline \multicolumn{6}{|l|}{ Stromal expression } \\
\hline SS 0 & $0(0)$ & $27(31.8)$ & $0(0)$ & $0(0)$ & $0(0)$ \\
\hline SS 1 & $27(31.8)$ & $54(63.5)$ & $66(77.7)$ & $66(77.7)$ & $59(69.4)$ \\
\hline SS 2 & $57(67.1)$ & $4(4.71)$ & $19(22.3)$ & $19(22.3)$ & $26(30.6)$ \\
\hline SS 3 & $1(1.2)$ & $0(0)$ & $0(0)$ & $0(0)$ & $0(0)$ \\
\hline \multicolumn{6}{|l|}{ Tumour blood vessel expression } \\
\hline BVS 0 & $0(0)$ & $21(24.7)$ & $0(0)$ & $0(0)$ & $0(0)$ \\
\hline BVS 1 & $13(15.3)$ & $50(58.8)$ & $30(35.3)$ & $10(11.8)$ & $47(55.3)$ \\
\hline BVS 2 & $69(81.2)$ & $14(16.5)$ & $55(64.7)$ & $75(88.2)$ & $38(44.7)$ \\
\hline BVS 3 & $3(3.5)$ & $0(0)$ & $0(0)$ & $0(0)$ & $0(0)$ \\
\hline
\end{tabular}

localisation of these proteins was cytoplasmic (Fig. 2a and b). For RhoA and Cdc42, co-expression in the cytoplasm and the nucleus was observed (70 cases, $82.35 \%$, and 6 cases, $7.1 \%$, respectively) (Fig. 2c and d). Immunostaining of RhoB revealed an additional sub-cellular localisation in the cellular membrane (14 cases, $16.47 \%$ ) and the perinuclear space (24 cases, $28.24 \%$ ) (Fig. 2e and f). Similar to the ERM protein family (i.e., ezrin and moesin), RhoA, RhoB and Cdc42 immunoreactivity was also observed in the stromal compartment of the breast tumour samples and its blood vessels (Table 3 ).

3.3 Relationship between ERM/Rho expression and status of steroid receptors and HER-2 reactivity

No relationship was observed between ERM/Rho protein expression and ER, PgR or HER-2 reactivity in the breast cancer specimens (Table 4). Interestingly, however, we observed a significant correlation between a decreased ezrin expression (BVS 0, 1, 2 versus BVS 3 ) and a positive PgR status in the tumour blood vessels $(P=0.028)$. In addition, we observed a correlation between low ezrin expression and HER-2 over-expression in the tumour blood vessels. This latter observation, however, was not statistically significant $(P=0.059)$.
3.4 Relationship between ezrin and moesin expression and clinicopathological parameters

Analysis of ezrin and moesin protein expression and patient clinicopathological parameters revealed significant correlations between protein expression and the presence of lymph node metastases in the tumour compartments $(P=0.047$ and $P=0.038$, respectively). We also observed a correlation between an increased ezrin immunoreactivity and the UICC stage (IIA versus IIB). This latter correlation was, however, not statistically significant. No significant relationships between ezrin or moesin expression and grading, tumour size and age at diagnosis were observed (Table 4). Notably, nuclear expression of ezrin was observed in $47.22 \%$ of the cases with lymph node metastases, compared to $18.37 \%$ in patients without nodal metastases $(P=0.004)$. We also found that nuclear expression of ezrin was associated with grade 3 (G3) breast cancer tumours $(P=0.015)$, thus confirming a potential role of nuclear ezrin immunodistribution in tumour development. No significant associations were observed between ezrin and moesin expression in the stromal compartments and tumour blood vessels and clinicopathological parameters, although a weak correlation between enhanced immunoreactivity of ezrin in the stroma and the postmenopausal status of patients was noted $(P=0.042)$ (Table 4$)$. 
Fig. 2 Immunohistochemistry of Rho proteins. a cytoplasmic localisation of RhoA (IRS $12, \times 200$, haematoxylin); b cytoplasmic localisation of Cdc42 (IRS 12, ×100, haematoxylin); c nuclear localisation of RhoA (IRS $9, \times 100$, haematoxylin); d nuclear localisation of $\mathrm{Cdc} 42$ (IRS 8, ×200, haematoxylin); e membranous localisation of RhoB (IRS 8, ×100, haematoxylin); f perinuclear localisation of RhoB (IRS $9, \times 100$, haematoxylin)
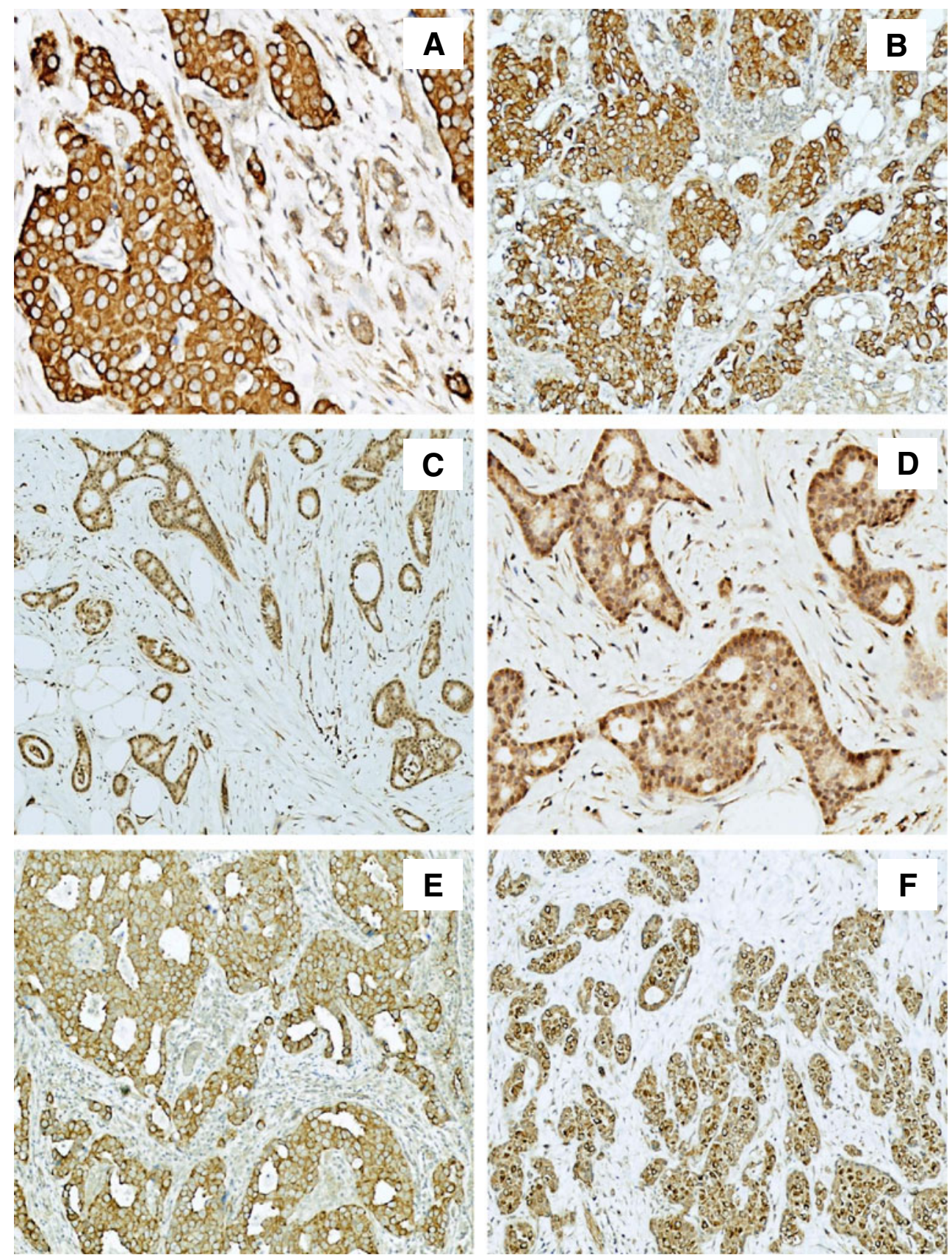

3.5 Relationship between RhoA, RhoB and Cdc42 expression and clinicopathological parameters

Over-expression of RhoA, RhoB and $\mathrm{Cdc} 42$ in the tumours was found to be associated with the presence of lymph node metastases $(P=0.024, P=0.004$ and $P=$ 0.047 , respectively). Additionally, we observed a statistically significant correlation between elevated RhoB expression and stage IIB according to the UICC ( $P=$ $0.033)$. Additional analyses of the sub-cellular distribution of RhoA revealed that a nuclear localisation was associated with a lack of nodal metastases $(P=0.008)$ and low grading $(P=0.055)$. Similarly, the nuclear topography of $\mathrm{Cdc} 42$ and perinuclear localisation of RhoB were both correlated with a lack of lymph node metastases in univariate analyses $(P=0.048$ and $P=$ 0.001 , respectively). No significant relationships were observed between the expression of RhoA, RhoB and $\mathrm{Cdc} 42$ and other clinicopathological parameters, including tumour size, grade, age at diagnosis and menopausal status (Table 4).

Expression analyses of the Rho proteins in the stromal compartments and tumour blood vessels in the $\mathrm{BC}$ specimens revealed intriguing findings. Notably, decreased reactivity of 
Table 4 Correlation between ERM/Rho expression and clinicopathological parameters

\begin{tabular}{|c|c|c|c|c|c|c|c|c|c|c|}
\hline & \multicolumn{10}{|c|}{ CLINICOPATHOLOGICAL PARAMETERS } \\
\hline & ER & $\operatorname{PgR}$ & HER-2 & $\begin{array}{l}\text { Tumour } \\
\text { size }(\mathrm{pT})\end{array}$ & $\begin{array}{l}\text { Nodal } \\
\text { metastases }(\mathrm{N}+)\end{array}$ & TNM (UICC) & Grading & $\operatorname{Age}^{\mathrm{a}}$ & $\begin{array}{l}\text { Menopause } \\
\text { status }\end{array}$ & $\begin{array}{l}\text { Adjuvant } \\
\text { CHT }\end{array}$ \\
\hline \multicolumn{11}{|l|}{ EZRIN } \\
\hline $\mathrm{IRS}^{\mathrm{b}}$ & 0.337 & 0.118 & 0.462 & 0.151 & 0.047 & 0.058 & 0.186 & 0.115 & 0.072 & 0.004 \\
\hline Membranous ${ }^{\mathrm{c}}$ & 0.527 & 0.091 & 0.382 & 0.923 & 0.383 & 0.961 & 0.523 & 0.582 & 0.872 & 0.665 \\
\hline Nuclear $^{\mathrm{c}}$ & 0.070 & 0.170 & 0.646 & 0.133 & 0.004 & 0.730 & 0.015 & 0.964 & 0.524 & 0.080 \\
\hline Stromal $(\mathrm{SS})^{\mathrm{c}}$ & 0.441 & 0.441 & 0.560 & 0.354 & 0.947 & 0.113 & 0.575 & 0.376 & 0.042 & 0.132 \\
\hline Blood vessels $(\mathrm{BVS})^{\mathrm{c}}$ & 0.117 & 0.028 & 0.059 & 0.092 & 0.124 & 0.470 & 0.630 & 0.738 & 0.415 & 0.507 \\
\hline \multicolumn{11}{|l|}{ MOESIN } \\
\hline $\mathrm{IRS}^{\mathrm{b}}$ & 0.944 & 0.644 & 0.473 & 0.315 & 0.038 & 0.460 & 0.770 & 0.812 & 0.748 & 0.884 \\
\hline Stromal $(\mathrm{SS})^{\mathrm{c}}$ & 0.354 & 0.236 & 0.252 & 0.578 & 0.375 & 0.177 & 0.344 & 0.346 & 0.480 & 0.483 \\
\hline${\text { Blood vessels }(B V S)^{c}}^{c}$ & 0.588 & 0.173 & 0.083 & 0.606 & 0.390 & 0.541 & 0.252 & 0.531 & 0.390 & 0.488 \\
\hline \multicolumn{11}{|l|}{ RhoA } \\
\hline $\mathrm{IRS}^{\mathrm{b}}$ & 0.730 & 0.928 & 0.928 & 0.292 & 0.024 & 0.845 & 0.093 & 0.528 & 0.691 & 0.333 \\
\hline Nuclear $^{\mathrm{c}}$ & 0.463 & 0.243 & 0.437 & 0.092 & 0.008 & 0.561 & 0.055 & 0.517 & 0.306 & 0.001 \\
\hline Stromal $(\mathrm{SS})^{\mathrm{c}}$ & 0.298 & 0.527 & 0.061 & 0.644 & 0.011 & 0.606 & 0.394 & 0.400 & 0.155 & 0.038 \\
\hline Blood vessels $(\mathrm{BVS})^{\mathrm{c}}$ & 0.398 & 0.601 & 0.414 & 0.531 & 0.149 & 0.268 & 0.351 & 0.587 & 0.259 & 0.191 \\
\hline \multicolumn{11}{|l|}{ RhoB } \\
\hline $\mathrm{IRS}^{\mathrm{b}}$ & 0.932 & 0.232 & 0.990 & 0.690 & 0.004 & 0.033 & 0.795 & 0.182 & 0.078 & 0.123 \\
\hline Membranous ${ }^{\mathrm{c}}$ & 0.482 & 0.272 & 0.167 & 0.481 & 0.065 & 0.603 & 0.603 & 0.478 & 0.524 & 0.482 \\
\hline Perinuclear $^{\mathrm{c}}$ & 0.011 & 0.038 & 0.110 & 0.422 & 0.001 & 0.522 & 0.344 & 0.346 & 0.165 & 0.028 \\
\hline Stromal $(\mathrm{SS})^{\mathrm{c}}$ & 0.133 & 0.527 & 0.017 & 0.644 & 0.477 & 0.200 & 0.200 & 0.210 & 0.155 & 0.568 \\
\hline${\text { Blood vessels }(B V S)^{c}}^{c}$ & 0.425 & 0.425 & 0.678 & 0.221 & 0.309 & 0.221 & 0.067 & 0.489 & 0.305 & 0.479 \\
\hline \multicolumn{11}{|l|}{ Cdc42 } \\
\hline IRS $^{\mathrm{b}}$ & 0.446 & 0.241 & 0.247 & 0.787 & 0.047 & 0.804 & 0.313 & 0.993 & 0.253 & 0.671 \\
\hline Nuclear $^{\mathrm{c}}$ & 0.614 & 0.386 & 0.690 & 0.526 & 0.048 & 0.563 & 0.181 & 0.293 & 0.489 & 0.313 \\
\hline Stromal $(\mathrm{SS})^{\mathrm{c}}$ & 0.504 & 0.147 & 0.510 & 0.537 & 0.152 & 0.053 & 0.924 & 0.087 & 0.018 & 0.187 \\
\hline${\text { Blood vessels }(B V S)^{c}}^{c}$ & 0.210 & 0.572 & 0.322 & 0.384 & 0.377 & 0.101 & 0.379 & 0.016 & 0.026 & 0.226 \\
\hline
\end{tabular}

${ }^{\text {a }} P$ value of Spearman's rank correlation

${ }^{\mathrm{b}} P$ value of $\chi^{2}$

${ }^{\mathrm{c}} P$ value of Fisher's test

Statistically significant results ( $P$ values) are underlined with bold text

RhoA in the tumour stromal compartment was correlated with the presence of lymph node metastases $(P=$ 0.011). Furthermore, the low level of Cdc42 observed in the tumour stromal compartment was correlated with a postmenopausal status $(P=0.018)$. In keeping with this latter finding, elevated $\mathrm{Cdc} 42$ reactivity in the tumour blood vessels was significantly correlated with the premenopausal status of patients $(P=0.026)$. Older women were characterised by a decreased expression of Cdc42 in the tumour blood vessels (Table 4).

3.6 Ezrin and moesin immunoreactivity and patient survival

Survival analyses did not reveal any prognostic significance of ezrin and moesin protein expression in BC patients. Also, no impact of the IRS of both proteins and their sub-cellular localisations on cancer-specific overall survival (CSOS) and disease-free survival (DFS) was noted (Fig. 3a).

3.7 RhoA, RhoB and Cdc42 immunoreactivity and patient survival

Survival analyses did reveal a significant association between the over-expression of RhoB in the stromal compartment and a better patient outcome $(P=0.0106)$ (Fig. 3c). Interestingly, this analysis did not reveal any impact of the sub-cellular distribution of RhoA. Correlation analyses between RhoA, RhoB and Cdc42 protein expression and patient survival did not reveal any statistically significant relationships (Fig. 3b and d). 

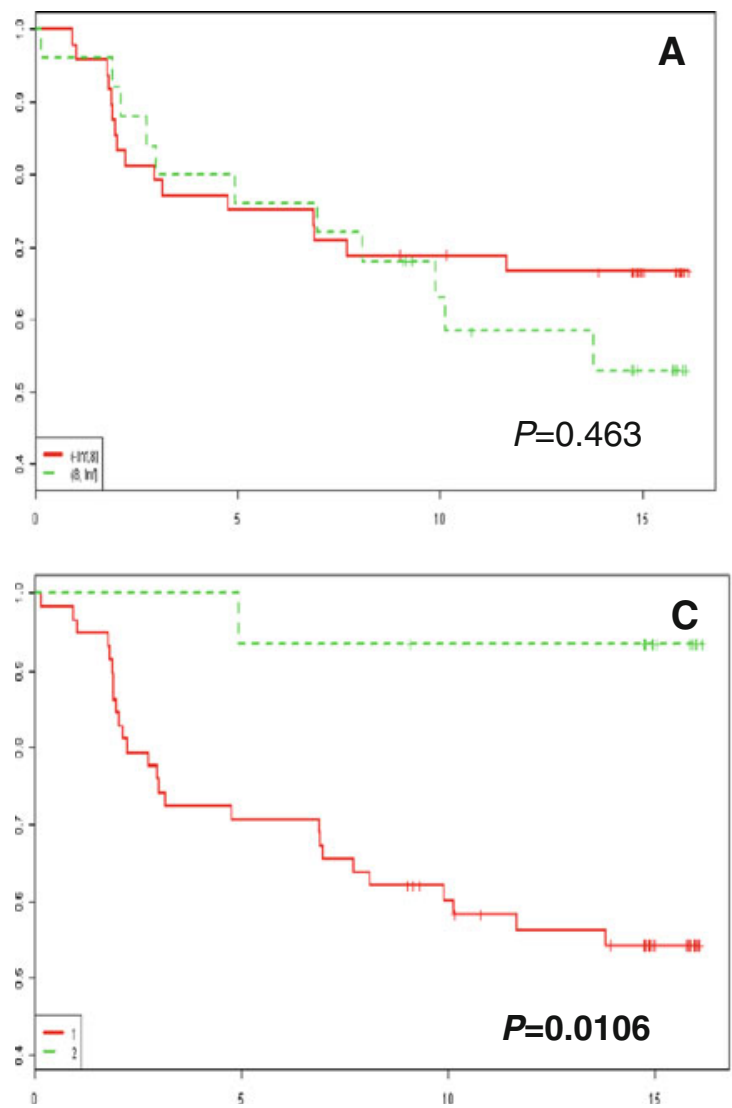

Fig. 3 Kaplan-Meier curves for cancer-specific overall survival (CSOS) and disease-free survival (DFS) and selected ERM/Rho immunohistochemical parameters. No significant differences in DFS and

\section{Discussion}

In this study, we investigated the expression of the ERM (ezrin, moesin) and Rho (RhoA, RhoB, Cdc42) protein families in a homogeneous group of patients with stage II invasive ductal breast cancer (BC). In addition, we assessed relationships between the sub-cellular localisation of these proteins, clinicopathological parameters and patient survival over a 15 year period. We also evaluated relationships between ERM/Rho reactivities in $\mathrm{BC}$ specimens and the status of steroid receptors and HER-2.

A correct sub-cellular localisation of the ERM and Rho proteins is essential for their normal function. The predominant localisation of all proteins analysed was cytoplasmic. In addition, our study revealed several other ERM/Rho subcellular topographies. Ezrin localisation was observed in both the nucleus and the cellular membrane, whereas moesin was only observed in the cytoplasm. RhoA and Cdc42 were characterised by a nuclear localisation, whereas RhoB was observed in the cellular membrane and the perinuclear zone. Interestingly, the nuclear expression of RhoA and $\mathrm{Cdc} 42$, and the perinuclear topography of RhoB, were strongly associated with a
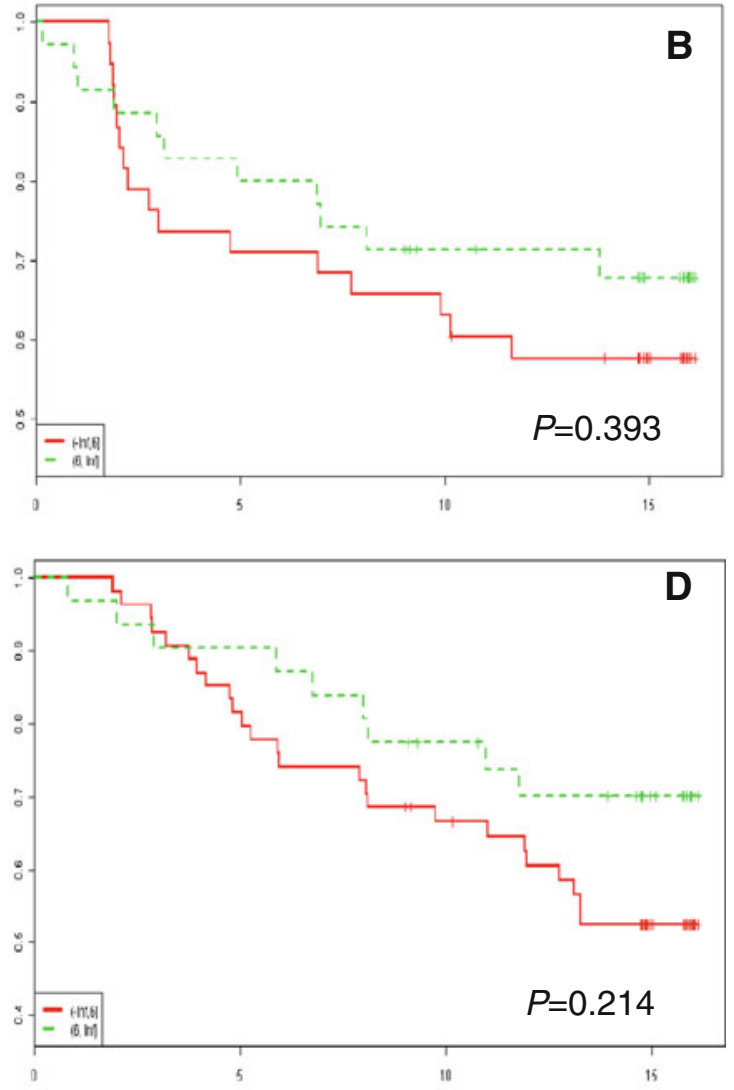

enhanced immunoreactivity of ezrin, RhoA and $\operatorname{Cdc} 42$ (a, b and d, respectively) are observed. $\mathbf{c}$ Patients with over-expression of RhoB in the stromal compartment exhibit an increased CSOS $(P=0.0106)$

lack of nodal metastases in our univariate analyses, but this was not reflected in the Kaplan-Meier survival analyses. Notably, however, the nuclear localisation of ezrin was associated with the presence of nodal metastases. We also observed that the nuclear localisation of ezrin was strongly correlated with grade $3 \mathrm{BC}$ tumours, thus confirming the potential role of nuclear ezrin immunodistribution in carcinogenesis.

To date, several sub-cellular ERM and Rho protein topographies have been described. For ezrin, cytoplasmic, membranous, nuclear and apical localisations have been reported [9-12]. Moesin was almost exclusively found to be located in the cytoplasm [23-25], with one study also reporting this protein to be located in the cellular membrane [24]. The latter was not observed in our study. The RhoA and RhoB sub-cellular localisation patterns reported in the literature are limited to the cytoplasm [26-29], with only one study reporting localisation in the cellular membrane [29]. Interestingly, Cdc42 expression has only been observed in the cytoplasm [30].

In this study, over-expression of ERM and Rho proteins in the tumour compartment of ductal $\mathrm{BC}$ was found to be significantly correlated with the presence of lymph node 
metastases, thus confirming the role of these molecules in $\mathrm{BC}$ progression. Similar results, indicating that cytoplasmic ezrin expression may act as a negative prognostic factor, were reported by Sarrio et al. [10], where the cytoplasmic expression of ezrin was strongly associated with lymph node metastases. In their study, Sarrio et al. specifically showed that differential ezrin localisations, rather than total protein levels, were correlated with a more aggressive behaviour of $\mathrm{BC}$ tumours. The change in ezrin distribution from the apical part of the membrane to either the complete membrane, or to the cytoplasm, has been associated with dedifferentiation of $\mathrm{BC}$ cells and unfavourable clinical features in invasive $\mathrm{BC}[10]$. It is worth noting that cytoplasmic ezrin is thought to exist in a dormant, inactive conformation, which is unable to associate with cellular membrane proteins and the actin cytoskeleton [7]. This, in turn, suggests that an altered ezrin sub-cellular distribution may be responsible for the de-regulation of ezrin-mediated cytophysiological processes. Intriguingly, we also observed complete membranous ezrin expression in 20 ductal BC cases $(23.53 \%)$. Because of the small patient cohort size, however, no significant prognostic value could be deduced. The possible biochemical mechanism, which may explain this phenomenon, relates to ezrin redistribution to the entire cellular membrane (not to the apical part), and may be influenced by external growth factors. Following stimulation, ezrin is present in an active form and, as such, can bind to membrane proteins and the actin cytoskeleton to create ezrin-rich cell membrane structures such as filopodia, which are strongly associated with enhanced motility, invasion and metastatic potential $[31,32]$. To the best of our knowledge, this is the first study to also describe a nuclear localisation of ezrin in BC cells (27/85 cases, $31.76 \%)$. Based on this location, ezrin might also act as a transcriptional factor. Consistent with our results, Charafe-Jauffret et al. [23] reported immunoreactivity of moesin in the cytoplasm of $\mathrm{BC}$ cells. Interestingly, they also observed moesin expression in the stromal compartment and tumour blood vessels, but the significance of this expression was not investigated in detail.

Rho family proteins are involved in the regulation of many different cellular processes, including migration, cell cycle progression, exocytosis and apoptosis. RhoA, RhoB and $\mathrm{Cdc} 42$ play a crucial role in the development of cancer, being main factors responsible for cellular motility and loss of adhesion. Expression of these proteins has been studied in many cancers, and various relationships have been revealed that clearly demonstrate the important role of Rho GTPases in carcinogenesis [26-28, 33, 34]. In our study, over-expression of RhoA, RhoB and Cdc42 was found to be strongly associated with the presence of lymph node metastases. Additionally, we observed a statistically significant correlation between an increased immunoreactivity of RhoB and stage IIB BC according to the UICC. Similar results on over-expression of Rho GTPases in breast cancer were reported by Fritz et al. [35]. In this latter study, RhoA, RhoB and $\mathrm{Cdc} 42$ protein levels were significantly elevated in $\mathrm{BC}$ samples compared to healthy breast tissues originating from the same individuals [35]. Intriguingly, RhoA and RhoB reactivity was found to significantly increase with the proliferation index and grading of the tumours, but the authors did not correlate Rho GTPase expression with clinicopathological parameters, such as nodal status or patient survival. In oesophageal squamous cell carcinoma, RhoA over-expression has significantly been correlated with the TNM stage, lymphatic and blood vessel invasion [27]. Moreover, 5-year survival rates were found to be lower in patients with enhanced reactivity of RhoA. A prognostic value of RhoA has also been observed in hepatocellular carcinoma, where a strong expression predicts a shorter survival rate [28]. Interestingly, Dittert et al. [26] revealed that RhoA expression in ductal pancreatic adenocarcinoma does not correlate with TNM classification, and that overexpression of this protein is strongly associated with a better prognosis. Furthermore, they observed stromal expression of RhoA, but did not correlate this with clinicopathological parameters. According to Pan et al. [36], a high expression of RhoA mRNA was significantly associated with advanced TNM classification and a low grade of histological differentiation. However, RhoB and $C d c 42$ mRNA levels did not correlate with the clinicopathological parameters studied. In retinoblastoma it was found that $\mathrm{Cdc} 42$ reactivity, measured by immunohistochemistry and Western blotting, had no prognostic value, and that over-expression of $\mathrm{Cdc} 42$ did not correlate with tumour invasion or histological differentiation [37]. In our study, an elevated expression of $\mathrm{Cdc} 42$ was found to be associated with nodal metastases, but qualitative analyses revealed paradoxical results, i.e., nuclear localisation of $\mathrm{Cdc} 42$ served as a good prognostic factor.

In summary, we report a first integrated expression analysis of different ERM and Rho protein family members in ductal breast cancer. ERM/Rho immunoprofiles and detailed sub-cellular localisation patterns may facilitate the prediction of lymph node metastases in ductal BC patients. No relationships were found between ERM/Rho expression and ER, PgR or HER-2 reactivity in BC cells. Also, survival analyses did not reveal a prognostic significance of ERM/Rho protein expression, except for RhoB overexpression in the stromal compartment of tumours, which was found to be associated with a better patient survival. Clearly, additional studies on extended patient cohorts are required to confirm our findings. Also, additional studies are needed to firmly establish the clinicopathological implications of ezrin, moesin, RhoA, RhoB and Cdc42 expression in breast carcinogenesis. 


\section{Conflict of interest None to declare.}

Sources of support This study was supported by Grant no. 0638/B/P01/2008/35 from the Ministry of Science and Higher Education (Poland) and by Grant no. ST-594 from Wroclaw Medical University (Poland)

Open Access This article is distributed under the terms of the Creative Commons Attribution License which permits any use, distribution, and reproduction in any medium, provided the original author(s) and the source are credited.

\section{References}

1. A. Jemal, F. Bray, M.M. Center, J. Ferlay, E. Ward, D. Forman, Global cancer statistics. CA Cancer J. Clin. 61, 69-90 (2011)

2. P.L. Fitzgibbons, D.L. Page, D. Weaver et al., Prognostic factors in breast cancer. College of American Pathologists Consensus Statement 1999. Arch. Pathol. Lab. Med. 124, 966-978 (2000)

3. P. Surowiak, P. Dziegiel, M. Zabel, R. Matkowski, J. Kornafel, Prognostic value of immunocytochemical estimation of estrogen receptor (ER) and of pS2 estrogen-dependent protein in cells of mammary ductal carcinoma. Analysis of five-year course of the disease. Folia Histochem. Cytobiol. 39, 143-144 (2001)

4. J.S. Ross, J.A. Fletcher, The HER-2/neu oncogene in breast cancer: prognostic factor, predictive factor, and target for therapy. Stem Cells 16, 413-428 (1998)

5. A. Goldhirsch, W.C. Wood, A.S. Coates, R.D. Gelber, B. Thurlimann, H.J. Senn, Strategies for subtypes - dealing with the diversity of breast cancer: highlights of the St Gallen International Expert Consensus on the Primary Therapy of Early Breast Cancer 2011. Ann. Oncol. 22, 1736-1747 (2011)

6. R.G. Fehon, A.I. McClatchey, A. Bretscher, Organizing the cell cortex: the role of ERM proteins. Nat. Rev. Mol. Cell Biol. 11, 276-287 (2010)

7. A. Bretscher, K. Edwards, F.G. Fehon, ERM proteins and merlin: integrators at the cell cortex. Nat. Rev. Mol. Cell Biol. 3, 586-599 (2002)

8. V. Niggli, J. Rossy, Ezrin/radixin/moesin: versatile controllers of signaling molecules and of the cortical cytoskeleton. Int. J. Biochem. Cell Biol. 40, 344-349 (2008)

9. B. Bruce, G. Khanna, L. Ren, G. Landberg, K. Jirström, C. Powell et al., Expression of cytoskeleton linker protein ezrin in human cancers. Clin. Exp. Metastasis 24, 69-78 (2007)

10. D. Sarrio, S.M. Rodriguez-Pinilla, A. Dotor, F. Calero, D. Hardisson, J. Palacios, Abnormal ezrin localization is associated with clinicopathological features in invasive breast carcinomas. Breast Cancer Res. Treat. 98, 71-79 (2006)

11. A. Elzagheid, E. Korkeila, R. Bendardaf et al., Intense cytoplasmic ezrin immunoreactivity predicts poor survival in colorectal cancer. Hum. Pathol. 39, 1737-1743 (2008)

12. A. Valdman, X. Fang, S.T. Pang et al., Ezrin expression in prostate cancer and benign prostatic tissue. Eur. Urol. 48, 852-857 (2005)

13. F.M. Vega, A.J. Ridley, Rho GTPases in cancer cell biology. FEBS Lett. 582, 2093-2101 (2008)

14. A.P. Wheeler, A.J. Ridley, Why three Rho proteins? RhoA, RhoB, RhoC, and cell motility. Exp. Cell Res. 301, 43-49 (2004)

15. M. Huang, G.C. Prendergast, RhoB in cancer suppression. Histol. Histopathol. 21, 213-218 (2006)

16. S.D. Merajver, S.Z. Usmani, Multifaceted role of Rho proteins in angiogenesis. J. Mammary Gland Biol. Neoplasia 10, 291-298 (2005)
17. L.H. Sobin, C. Wittekind, TNM Classification of Malignant Tumours, 5th edn. (Wiley-Liss INC., New York, 2002)

18. C.W. Elston, I.O. Ellis, Pathological prognostic factors in breast cancer. The value of histological grade in breast cancer: experience from a large study with long-term follow-up. Histopathology 5, 403-410 (1991)

19. A. Halon, V. Materna, M. Drag-Zalesinka et al., Estrogen receptor alpha expression in ovarian cancer predicts longer overall survival. Pathol. Oncol. Res. 17, 511-518 (2011)

20. A. Halon, E. Nowak-Markwitz, A. Maciejczyk et al., Loss of estrogen receptor beta expression correlates with shorter overall survival and lack of clinical response to chemotherapy in ovarian cancer patients. Anticancer. Res. 31, 711-718 (2011)

21. K. Szczuraszek, A. Halon, V. Materna et al., Elevated YB-1 expression is a new unfavorable prognostic factor in nonHodgkin's lymphomas. Anticancer. Res. 31, 2963-2970 (2011)

22. W. Remmele, H.E. Stenger, Recommendation for uniform definition of an immunoreactive score (IRS) for immunohistochemical estrogen receptor detection (ER-ICA) in breast cancer tissue. Pathologe 8, 138-140 (1987)

23. E. Charafe-Jauffret, F. Monville, F. Bertucci et al., Moesin expression is a marker of basal breast carcinomas. Int. J. Cancer 121, 1779-1785 (2007)

24. H. Kobayashi, J. Sagara, H. Kurita et al., Clinical significance of cellular distribution of moesin in patients with oral squamous cell carcinoma. Clin. Cancer Res. 10, 527-580 (2004)

25. M. Tokunou, T. Niki, Y. Saitoh et al., Altered expression of the ERM proteins in lung adenocarcinoma. Lab. Investig. 80, 1643-1650 (2000)

26. D.D. Dittert, C. Kielisch, I. Alldinger et al., Prognostic significance of immunohistochemical RhoA expression on survival in pancreatic ductal adenocarcinoma: a high-throughput analysis. Hum. Pathol. 39, 1002-1010 (2008)

27. A. Faried, M. Nakajima, M. Sohda et al., Correlation between RhoA overexpression and tumour progression in esophageal squamous cell carcinoma. Eur. J. Cancer Surg. 31, 410-414 (2005)

28. X.R. Li, F. Ji, J. Ouyang et al., Overexpression of RhoA is associated with poor prognosis in hepatocellular carcinoma. Eur. J. Surg. Oncol. 32, 1130-1134 (2006)

29. Y. Liu, Y. Wang, Y. Zhang et al., Abnormal expression of p120-catenin, E-cadherin, and small GTPases is significantly associated with malignant phenotype of human lung cancer. Lung Cancer 63, 375-382 (2009)

30. G. Goteri, A. Ciavattiani, G. Laucarini et al., Expression of motilityrelated molecule $\mathrm{Cdc} 42$ in endometrial tissue in women with adenomyosis and ovarian endometriomata. Fertil. Steril. 86, 559-565 (2006)

31. A. Gautreau, D. Louvard, M. Arpin, Morphogenic effects of ezrin require a phosphorylation-induced transition from oligomers to monomers at the plasma membrane. J. Cell Biol. 150, 193-203 (2000)

32. Z. Chen, A. Fadiel, Y. Feng et al., Ovarian epithelial carcinoma tyrosine phosphorylation, cell proliferation, and ezrin translocation are stimulated by interleukin 1alpha and epidermal growth factor. Cancer 92, 3068-3075 (2001)

33. T. Kamai, T. Yamanishi, H. Shirataki et al., Overexpression of RhoA, Rac1, and Cdc42 GTPases is associated with progression in testicular cancer. Clin. Cancer Res. 10, 4799-4805 (2004)

34. S. Rihet, P. Vielh, J. Camonis et al., Mutation status of genes encoding RhoA, Rac1, and Cdc42 GTPases in a panel of invasive human colorectal and breast tumors. J. Cancer Res. Clin. Oncol. 127, 733-738 (2001)

35. G. Fritz, C. Brachetti, F. Bahlmann et al., Rho GTPases in human breast tumours: expression and mutation analyses and correlation with clinical parameters. Br. J. Cancer 87, 635-644 (2002)

36. Y. Pan, F. Bi, N. Liu et al., Expression of seven main Rho family members in gastric carcinoma. Biochem. Biophys. Res. Commun. 315, 686-691 (2004)

37. M. Adithi, N. Venkatesan, M. Kandalam et al., Expression of Rac1, Tiam1 and Cdc42 in retinoblastoma. Exp. Eye Res. 83, 1446-1452 (2006) 